\title{
FRAMEWORKS TO IDENTIFY BEST PRACTICES AT THE ORGANIZATION LEVEL: AN ANALYSIS
}

\section{Gerardo Luís ANGULO CUENTAS 1 , Jaime Alberto CAMACHO PICO², Astrid JAIME ARIAS ${ }^{3}$}

\author{
${ }^{1}$ Industrial Engineering Department, Engineering Faculty, University of Magdalena, \\ Carrera 32 No. 22-08, Santa Marta, Colombia \\ ${ }_{2}^{2}$ Industrial Engineering School, Physical Mechanical Engineering Faculty, \\ Industrial University of Santander, Carrera 27 Calle 9, Bucaramanga, Colombia \\ ${ }^{3}$ Knowledge Transfer Office, Industrial University of Santander, Carrera 27 Calle 9, \\ Bucaramanga, Colombia \\ E-mails: ${ }^{1}$ gerardoangulo@unimagdalena.edu.co (corresponding author); \\ 2jcamacho@uis.edu.co; ${ }^{3}$ dirconocim@uis.edu.co
}

Received 19 June 2012; accepted 30 October 2012

\begin{abstract}
This paper identifies and analyzes the existing frameworks for supporting the identification and classification of best practices at an organization level.

The major contribution of this paper is the proposition and application of three criteria for analyzing, comparing and classifying the four identified frameworks. The first criterion is based on structural complexity, facilitates defining the scope of initiatives to identify best practices. The second one, based on the framework orientation, permits choosing a framework depending of the type of studied processes. While the third criterion based on organizational levels, permits comparing and classifying the frameworks in terms of the strategic, tactic or operational issues.

In conclusion are outlined important aspects in the selection of a framework according to two proposed scenarios: the best practices searching on Organizations of Excellence and the best practices searching on organizations with a similar business model.
\end{abstract}

Keywords: best practices, good practices, recommended practices, identification, frameworks, benchmarking.

JEL Classification: L23, L25, M10.

\section{Introduction}

A practice can be understood as the way an organization develops a specific process (Cf. O'Leary 2007). Those practices that have been widely recognized over time as excellent approaches for many organizations, and recommended by a large number of practitioners or experts to adopt for successful results, are viewed as good or the best practices (Xu, Yeh 2010). A best practice can be defined as a process that is better at 
delivering a particular result than any other. The word "better"can also be understood in two different ways: effectiveness/performance, i.e. whether the right things are done, and efficiency, i.e. whether the things done are done properly (Bergek, Norrman 2008). The best practices can be identified and learned from many sources such as industrial experiences (e.g. practitioners), consulting experiences (e.g. experts), advanced information systems (e.g. ERP systems), and knowledge base (Xu, Yeh 2010).

The identification along with the transfer practices, are extremely difficult processes and there is no consensus among consultants as to the appropriate methods to identify the best practices (Wellstein, Kieser 2011). Laugen et al. (2005), assume that the best performing companies must be the ones deploying the best practices. Their assumption focuses on manufacturing practices, performance and defining the best practices by which practices they are used by. This having significant effect on the performance of the best performing companies. While award-winning organizations are shown as a model of how an organization should be managed, Harrington (2004) suggests what may be good for one organization can actually be disastrous for another, based on an international management practice database.

As it can be observed there is a variety of approaches and concepts related with best Practices Identification at the organization level. Consequently, one wonders where and how best practices can be found. In this paper we do not pretend to answer these questions, because we want to provide new elements for researchers and practitioners to make better decisions in choosing an approach or framework to identify best practices at the organization level. For this reason, this paper analyzes existing frameworks that can support this process and proposes three classifications for them.

This paper is structured in the following way. First, we present the Method section, where we expose the way this work has been carried out. Next, we describe the existing frameworks that can support the identification of best practices and some applications of these frameworks. Subsequently, we propose and discuss three classifications for the identified frameworks. These classifications are proposed as criteria to compare them and determine the frameworks' suitability to a particular research. Finally, we present the conclusions of the research and propose directions for further research.

\section{Method}

As first step we searched for articles published in journals indexed in Thomson Reuters' Web of Science under three initial criteria: a) contains the words "best" and "practices" in the title; b) Published during the period January 2001 to October 2011; c) Indexed in Science Citation Index Expanded (SCI-EXPANDED) or Social Sciences Citation Index (SSCI). As a result of this process, we obtained 2,051 articles.

The second step was refining the initial search by the Web of Science Categories. We selected the articles in the next five categories: Management, Economics, Business, Engineering Industrial and Business Finance. We eliminated articles that did not belong in these five categories. As a result, this left us with a set of 211 articles. 
The third step consisted in reading journal names, titles and abstracts of the articles' set. We found that most of the papers were studies or works in which the identification or description of organizations' best practices was not the subject of the analysis. To select relevant literature, we adopted two inclusion criteria. First, a paper must deal with the best practices identification concept as main subject and in an explicit way. Second, a paper also must refer to the best practices identification at the organization (company or firm) level. As a result, 172 papers did not fit these criteria and were eliminated. This left us with a final sample of 39 publications.

The analysis of these papers revealed two approaches for identifying best practices at the company level: The first approach was the process-focused. This consisted of studying a specific process from many organizations. The activities that had a direct relationship with a better performance of process being studied are considered best practices. The second approach was the organizational performance-focused approach which consisted in studying a group of organizations under excellence or high performance criteria. Then identifying what activities they complete in their different processes.

Most of papers analyzed (28 of 39) use the process-focused approach, while only four papers of the sample use the organizational performance-focused approach. Since the second approach has been the least explored, we decided to research further into available resources and additional literature related to this approach. The results and a proposed analysis are shown in the following sections.

\section{Frameworks to identify best practices at the organization level}

Before sorting a specific practice into the category of recommended, good, better or best, we considered it advisable to have a neutral approach. So, we proposed to do this by using the essence of the process. The essence of the process is the attribute, or set of attributes, that make a process what it fundamentally is. With which it has necessity, and without which it loses its identity. This set of properties defines a process as unique and different from others but also allow association with others in the same class. Frameworks and taxonomies of processes can be used for this purpose.

The following frameworks can be used for simultaneously studying several organizations in order to identify best practices. We found that the use of the criteria of the excellence model of the European Foundation for Quality Management (hereafter EFQM). As a North American equivalent, we propose the use of the Criteria for Performance Excellence of the Baldrige Performance Excellence Program (hereafter Baldrige) proposed by the National Institute of Standards and Technology (NIST).

As taxonomies of processes that provide value for the classification of best practices, O'Leary (2007) recognizes the APQC Process Classification Framework (hereafter APQC-PCF) proposed by the American Productivity \& Quality Center (APQC) and the Process Classification Framework proposed by Price Waterhouse Coopers (hereafter PWC-PCF).

Then we proceed to describe the aforementioned Frameworks. 


\subsection{The EFQM model and the Baldrige program criteria as frameworks for identifying best practices}

These frameworks share two objectives: they were designed for self-assessment in organizations and as the criteria for granting awards distinguished by its excellence.

\subsubsection{The EFQM excellence model}

Powered by EFQM (2012a 2012b, 2009), is a framework used by organizations in Europe and beyond, and it can be used as a diagnostic tool. The model is comprehensive and intended to be useful for organizations regardless of size or sector. First, it can be used to assess how they are, which allows you to identify strengths and potential weaknesses in performance across nine criteria. Second, to provide a common vocabulary and way of thinking about the organization that facilitates the effective communication of ideas, both within and outside the organization. Third, used to integrate existing and planned initiatives to avoid duplication and identify gaps. Finally, this framework is used to have a reference model for the basic structure of its management system.

The model comprises of a set of three integrated components: The Fundamental Concepts of excellence, the nine criteria and the tool RADAR.

The Fundamental Concepts of excellence proposes a set of underlying principles in order to achieve sustainable excellence in any organization. They consider eight key concepts in order to become an Organization of Excellence (hereafter OE): 1) Achieving balanced results: OEs meet their Mission and progress towards their Vision through planning and achieving a balanced set of results that meet both the short and long term needs of their stakeholders and, where relevant, exceed them. 2) Adding value for customers: OEs know that customers are their primary reason for existing and strive to innovate and create value for them, by understanding and anticipating their needs and expectations. 3) Leading with vision, inspiration and integrity: OEs have leaders who shape the future and make it happen, often acting as role models for values and ethics. 4) Managing by processes: OEs are managed through structured and strategically aligned processes that use fact-based decision making to create balanced and sustained results. 5) Succeding through people: OEs value their people and create a culture of empowerment for the balanced achievement of organizational and personal goals. 6) Nurturing creativity and innovation: OEs generate increased value and levels of performance through continual and systematic innovation by harnessing the creativity of their stakeholders. 7) Building partnerships: OEs seek, develop and maintain trusting relationships with various partners to ensure mutual success. 8) Taking responsibility for a sustainable future: OEs embed within their culture an ethical mind-set, clear values and the highest standards for organizational behavior. All of which enable them to strive for economic, social and ecological sustainability.

The nine criteria would allow people to understand the cause and effect relationships between what their organization does, the Enablers, and the Results that it achieves.

The Enablers comprise of: 1) Leadership: five sub-criteria for evaluating how organization's leaders: a) develop and deploy the organization's strategy and values, b) monitor 
and drive the improvement of the organization's management system and performance, c) engage with the stakeholders, d) reinforce a culture of excellence and e) manage organizational flexibility and change. 2) Strategy: four sub-criteria to verify in the strategy: a) stakeholder's needs, b) internal performance and capabilities, c) economic, societal and ecological sustainability, d) deployment way. 3) People: five sub-criteria for identifying how organization's people: a) support the strategy, b) develop their knowledge and capabilities; c) are involved and empowered, d) communicate throughout the organization, e) are rewarded, recognized and cared for. 4) Partnerships and Resources: five sub-criteria about are managed: a) Partners and suppliers for a sustainable benefit, b) Finances to secure sustained success, c) Buildings, equipment, materials and natural resources in a sustainable way, d) Technology to support the delivery of strategy, e) Information and knowledge to support effective decision making and build the organizational capability. 5) Processes, products and services: five sub-criteria to understand how the value is created when the processes, products and services are: a) designed, b) developed, c) promoted and marketed, d) produced, delivered and managed, e) customer's relationships are managed and enhanced.

On the other hand, the Results comprise: 6) Customer results: focusing the customers' perceptions about the organizations impact and their monitoring indicators. 7) People results: how the people perceive strategy's effectiveness and their monitoring indicators. 8) Society results: how the society perceives the social and environmental impact generated by the organization and their monitoring indicators. 9) Key results: the results delivered by the strategies deployment and their monitoring indicators.

The third component of the EFQM model is the RADAR tool: RADAR (results, approaches, deploy, assess and refine) was designed as a structured approach for evaluating the performance of an organization. It is also used as a scoring mechanism for the EFQM Excellence Award. The score relates to the evidence of the existence of effective implementation of the Enablers criteria. As well as the Enabler's effect, relevance, reliability and sustainability are all a part of the Results criteria. However, it is not the subject of this paper, so we will not delve into details.

Reviewing the literature on identification of best practices using the EFQM model, we highlight the following works.

Oakland et al. (2002) present illustrations of best practices under each of the EFQM's criteria, together with an analysis of how public and private sector successful organizations achieve total quality through feedback, learning and innovation. Also, how they have addressed the requirements of the EFQM's model to bring about excellent performance.

Osseo-Asare et al. (2005) identified and classified the leadership practices in 42 educational institutions in the UK. Through questionnaires, interviews and testing of hypotheses explored the critical factors of effective leadership, as well as describing the best practices of leadership. To classify practices, they define the categories of weak, good, best and excellent practices, based on efficiency and effectiveness of each one to achieve and sustain academic quality. 
McCarthy (2005), for investigating the differences in leadership practices between organizations in Germany and the UK, analyzed self-assessment documents (based on the standard of excellence) submitted to the EFQM and applied a survey to determine what practices were considered common practices and best practices. It is emphasized that the practices considered as best practices in the UK were not the most common practices.

Vouzas and Gotzamani (2005) researched ten organizations that they consider representative of Greece, all winners of the EFQM Excellence Award. In addition to success stories about implementing the model, they find problem areas that could be documented as malpractices and as such, which would also be another source of learning.

\subsubsection{The Baldrige performance excellence program criteria}

This program is a public-private initiative in the U.S.A., dedicated to promoting excellence in organizational performance. It aims to raise awareness about the importance of performance excellence in the conduct of the U.S. and the global economy. It has developed tools and criteria for evaluating organizations and training programs on organizational best practices. In order to recognize the efforts in organizational excellence, it grants the Malcolm Baldrige National Quality Award. The award criteria can be used to identify and classify best practices. There are three versions of these criteria: business and nonprofit organizations (NIST 2013a), education (NIST 2013b) and organizations for health care (NIST 2013c). We will be describing only the former because it is the most general and applicable to a larger number of organizations.

This framework includes seven categories, allows a quantitative assessment of excellence in an organization, the score for each category is as follows: 1) Leadership (120 points), 2) Strategic Planning (85 points), 3) Customer Focus (85 points), 4) Measurement, Analysis and knowledge Management (90 points), 5) Workforce Focus (85 points), 6) Operations Focus (85 points) and 7) Results (450 points). The total score is obtained by adding the scores achieved in each category for a maximum of 1000 points.

1) Leadership considers the leadership of senior management as well as governance and social responsibility. 2) Strategic Planning takes into account the development and implementation of the strategy. 3) Customer Focus is about how the customer's voice is heard and how to build relationships. 4) Measurement, Analysis and knowledge Management is related to how the organization improves its performance through the use of data and information at all levels and how it develops and manages its knowledge assets. 5) Workforce Focus comprises how to create an effective support environment for the workforce and how it engages the workforce to achieve organizational and personal success. 6) Operations Focus is understood how to design manage and improve work systems and key processes. 7) Results evaluate five items: the product performance and effectiveness of processes; performance on customer focus (satisfaction and customer relations); performance on workforce focus (skills, abilities, climate, commitment, satisfaction and leadership); performance of senior management and governance; and financial performance and market. 
We found only one study using this framework for identifying best practices. Saunders et al. (2007) analyzed seven organizations in New Zealand that were developing an improvement program based on the BALDRIGE model. The unit of analysis was the strategic initiative that the organization had developed, and they found more than 50 best practices from which they developed a framework for the deployment of the strategy in organizations.

\subsection{Process classification taxonomies as support for identifying best practices: the APQC Process Classification Framework (APQC-PCF) and the Price Waterhouse Coopers Process Classification Framework (PWC-PCF)}

From a common origin around 1992 (O'Leary 2007), these two frameworks have evolved into two separate frameworks. Therefore they share similarities in the different levels of classification, being more pronounced in the first level. This is where they classify processes as operating processes (both) and as management processes (PWC 2011) or as management and support services (APQC 2014).

\subsubsection{The APQC Process Classification Framework (APQC-PCF)}

It was originally designed (1992) as a taxonomy and a common language for APQC's member organizations in order to make comparisons and contrasting views on processes across different industries. Since then it has been tested for over 15 years by thousands of organizations from around the world (APQC 2014). In 2008, along with IBM, the APQC updated the generic model and developed some adaptations for eleven specific industries: aerospace and defense, automotive, banking, broadcasting, consumer products, education, electric utilities, petroleum downstream, petroleum upstream, pharmaceuticals and telecommunications. The generic framework is described in the following. They propose this descending hierarchy taxonomy:

$$
\text { Category }>\text { Process Group }>\text { Process }>\text { Activity. }
$$

Category: The highest level within the APQC-PCF is indicated by whole numbers. Operating processes are 1.0 Develop Vision and Strategy, 2.0 Develop and Manage Products and Services, 3.0 Market and Sell Products and Services, 4.0 Deliver Products and Services, 5.0 Manage Customer Service. Management and Support Services are 6.0 Develop and Manage Human Capital, 7.0 Manage Information Technology, 8.0 Manage Financial Resources, 9.0 Acquire, Construct, and Manage Property, 10.0 Manage Environmental Health and Safety (EHS), 11.0 Manage External Relationships, 12.0 Manage Knowledge, Improvement, and Change.

Process Group: Items with one decimal numbering. An example: 1.1 Define the business concept and long-term vision, is a process group within the category 1.0 Develop vision and strategy.

Process: Items with two decimal numberings are considered processes. An example, 1.1.3 Perform internal analysis is a process within the process group 1.1 Define the business concept and long-term vision. 
Activity: Items with three decimal numbering are considered activities within a process. An example: 1.1.3.1 Analyze organizational characteristics is considered an activity within the process 1.1.3 Perform internal analysis.

Within scientific literature, we did not find any work based on APQC-PCF for identifying best practices.

\subsubsection{The Price Waterhouse Coopers Process Classification Framework (PWC-PCF)}

Price Waterhouse Coopers used to use this framework to organize best practice tools and information. It contains 13 business processes that apply to almost any business. The first seven are operating processes that companies follow to develop and move products to the market. The last six processes are management and support processes that make it possible for the company to operate effectively (PWC 2011).

The PWC-PCF does not distinguish hierarchies among describing processes despite using two decimal places to define more specific processes. For example: Figure 1 shows that despite some processes being contained within others, all items are considered as processes, so it does not make distinctions as process groups, macro-processes or other classification.

In the PWC-PCF, the seven operating processes are: 1.0 Understand Markets And Customers, The process of analyzing market and customer information to create and maintain a competitive business position; 2.0 Develop Vision And Strategy, The process of preparing for future success with strategic plans that address both short and long-term business objectives; 3.0 Design Products And Services, The process of conceiving, designing, and delivering competitive products and services; 4.0 Market And Sell, The process of developing strategies and procedures to position products and services in the marketplace to achieve high levels of the market share; 5.0 Produce And Deliver Products And Services, The process of managing the production process, from resources and project management, to inventory and warehousing, ending with delivery and service support; 6.0 Produce And Deliver For Service Oriented Organizations; The process of managing the service delivery process, from resource and project management, to employee support and customer relations, finishing with delivery and quality assurance; and 7.0 Invoice And Service Customers, The process of billing customers for goods and

1.0. UNDERSTAND MARKETS AND CUSTOMERS: The process of analyzing market anil customer information to create and maintain a competitive business position.

1.1. Determine customer needs and wants: The process of identifying customer segments, critical incidents of customer contact, and customer needs in order to meet customer demands.

1.1.1. Capture and assess customer feedback: The process of eliciting, analyzing, and applying direct and indirect customer feedback to improve operational performance.

Fig. 1. Three levels of process understanding markets and customers in the PWC-PCF Source: PWC (2011). 
services rendered and providing after-sales support and problem resolution to retain customers and build loyalty.

On the other hand, the six management and support processes are 8.0 Develop And Manage Human Resources, The process of planning and monitoring issues pertaining to the workforce, including recruitment, hiring, compensation, administration of benefits, and training of employees; 9.0 Manage Information Resources And Technology, The process of ensuring timely and affordable access to state-of-the art information tools and technology; 10.0 Manage Financial And Physical Resources, The process of protecting resources and systems fundamental to the viability of the business; 11.0 Manage Environmental, Health, And Safety Issues, The process of fostering top-down, proactive EHS awareness and management to improve performance and achieve goodwill among key stakeholders; 12.0 Manage External Relationships, The process of ensuring the support and loyalty of key stakeholders through effective relationship management; and 13.0 Manage Improvement And Change, The process of ensuring that necessary changes in the work environment and business processes are well-understood and supported by solid transition plans.

Within scientific literature, we did not find any work based on PWC-PCF for identifying best practices.

\section{A proposal to classify these frameworks}

After reviewing these four frameworks, we proceeded to complete a comparative analysis of them. We propose three criteria: complexity, management-oriented or operationsoriented and organization levels.

\subsection{Complexity of the frameworks}

We consider that a framework can be more complex than others depending on the number of layers and elements present inside each of them. In each of the frameworks described one can observe that they have several levels or layers to categorize activities or processes (practices) that occur in an organization. Also, one can find many elements in each layer and they represent activities or processes (practices). Accordingly, all frameworks under analysis can be seen as tree structures, and be evaluated through structural complexity.

Westfall (2010), defining structural complexity metrics, affirms that the depth and width metrics focus on the complexity of the entire structure. This author defines depth as the number of levels of control, and width as the number of span of control, in the overall structure. Based on these concepts, this analysis understands depth and width as follows:

- Depth of a framework is the number of layers or levels (categories of practices) from the top to the bottom of the structure.

- Width of a framework is the maximum number of elements (practices) in the deepest layer.

We called layer 0 (zero) the initial dichotomy of each of the frameworks, three of them have explicit this layer: EFQM (enablers and results), APQC-PCF (operating processes 
and management and support services), PWC-PCF (operating processes and management processes). In the case of BALDRIGE, this framework does not have an explicit layer zero and we propose two categories (processes and results). According to the proposed metrics, it would have the following descending order of complexity:

$$
\text { APQC-PCF }>\text { PWC-PCF }>\text { EFQM }>\text { BALDRIGE. }
$$

We consider APQC-PCF as the most complex because it has five layers and in its deepest layer (Layer four) there are 14 elements to classify activities in an organization. PWC-PCF has four layers and six types of processes in its deepest layer (layer three). EFQM and Baldrige both have three layers and in their deepest layer (layer two) both have five elements. EFQM is considered more complex than Baldrige because in the top layer (layer one) there are nine elements and within Baldrige there are seven elements.

Structural complexity of the studied frameworks can be useful to define the scope of initiatives to identify best practices. The more complex frameworks (APQC-PCF and PWC-PCF) provide more resources to hierarchize and classify executed practices in any organization. According to their width and depth, these frameworks facilitate the study of a wide and diverse spectrum of organizational practices while at the same time, explore details about them. Although EFQM and Baldrige are less complex, they present the same width (five elements), and the same depth (three layers). For this reason, initiatives based on these frameworks could have a more limited scope than others based on APQC-PCF or PWC-PCF.

In Figure 2 it can be seen partially the analysis described.

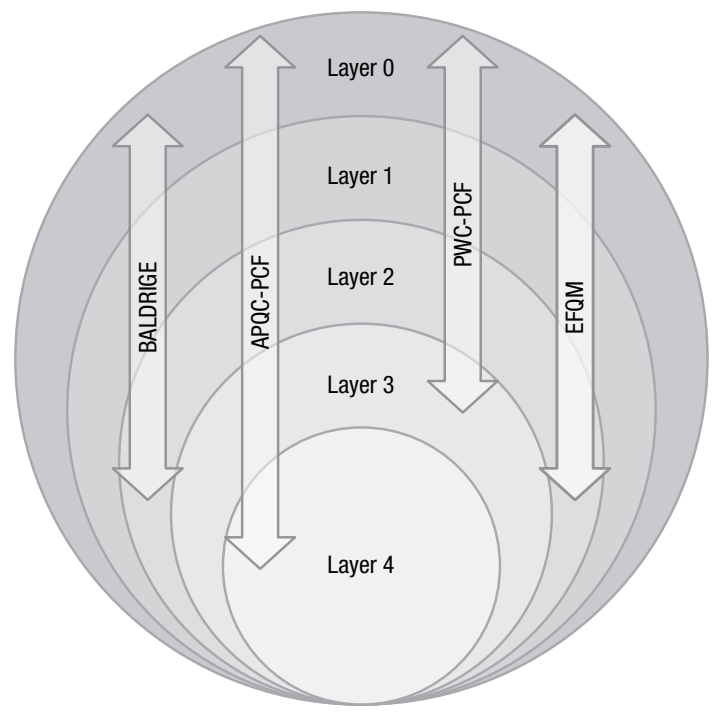

Fig. 2. The depth as dimension of complexity in the analyzed frameworks Source: created by authors. 


\subsection{The predominant orientation: management or operations}

All analyzed frameworks consider processes and activities related to both management and operational issues. However, when analyzing we will find marked orientations towards one of them (see Fig. 3).

EFQM and Baldridge are models that aim to develop or evaluate organizational excellence, so they focus their criteria in management and control. Both models prioritize areas such as leadership, strategy, people or work force, alliances, and customer relationships. However, above all of them, these models focus in the results, measurement and control.

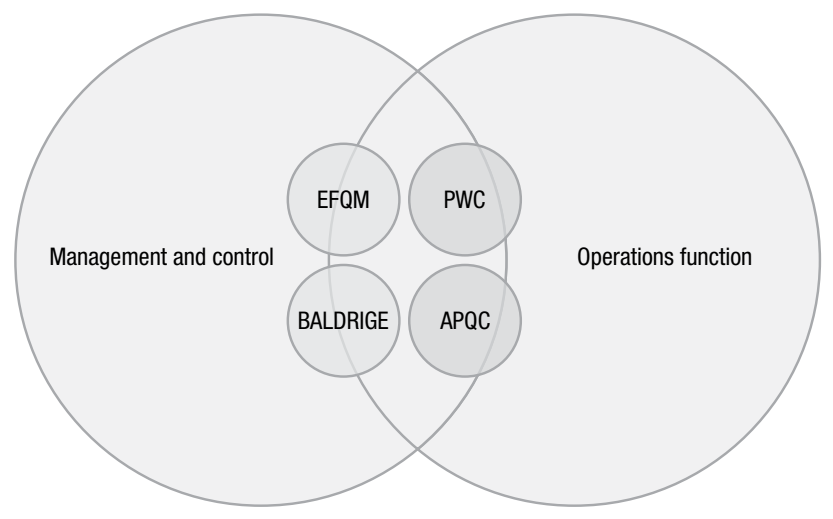

Fig. 3. Frameworks orientation. Management or operations Source: created by authors.

APQC-PCF and PWC-PCF have proportions relatively equal for the management and operations in layer one, but its strength is in the depth and detail on operational processes. They have more layers and elements in each layer in this type of process than EFQM and Baldridge.

Depending on the type of best practices to identify (operating or management processes) it can effect the choice of a framework. We suggest, APQC and PWC-PCF$\mathrm{PCF}$ for operational processes and EFQM and Baldrige for management and control processes.

\subsection{Organization levels}

Overall, it is possible to identify three levels in an organization: the corporate level, business unit and the functional, or operational, level (Verweire, Van den Berghe 2004). The studied frameworks are focused on the corporate level or the functional level, and fewer elements are located at that level of the business unit than in the others. Figure 4 shows the concentration of elements in each framework at different levels of an organization.

We recommend EFQM and Baldrige for the corporate level because both focus on issues such as leadership, strategy and the process of planning it, people or the work force, alliances, and customer focus. Comparatively, they pay little attention to pro- 
cesses, products and services in the case of EFQM and 'operations focus' in the case of Baldrige. So, they have been associated with lower concentrations at the business unit level as well as the functional level.

APQC-PCF and PWC-PCF (as shown in Fig. 4) have higher concentrations at the functional level, because both emphasize aspects such as the market and the sales of products and services, production and delivery of products and services, customer billing and support, financial management and property management, and information resources and technology among other criteria. However, both do consider, although with much lesser concentration, the development of vision and strategy, knowledge management, improvement as well as change. Due to this minimal focus, we don't really associate them to the corporate level.

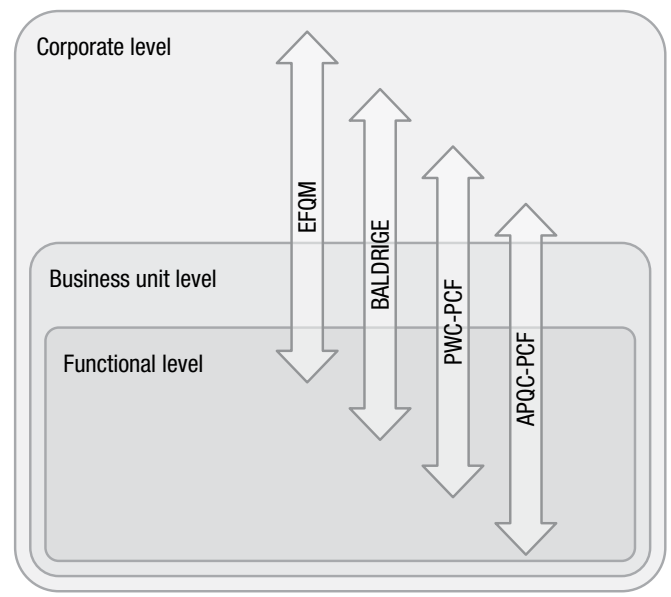

Fig. 4. Concentration of elements in each framework at different levels of an organization Source: created by authors.

This classification on the level within the organization may be useful for defining what best practices will be identified in as far as terms of the strategic, tactic or operational issues.

\section{Conclusions}

The main objective of the present research was to identify and analyze the existing frameworks for supporting the identification and classification of best practices at the organization level.

From a theoretical standpoint, this paper contributes by proposing and applying three criteria for analyzing, comparing and classifying the four identified frameworks: the EFQM excellence model, the Baldrige Criteria for Performance Excellence, the APQC's Process Classification Framework and the PWC Process Classification Framework. The first criterion, which is based on structural complexity, facilitates of defining the scope of initiatives to identify best practices. 
The more complex frameworks will permit a deeper exploration of the organizational levels, and a detailed way the processes or activities are developed inside (practices). The less complex frameworks would permit rapid checking of best practices but with lower precision. The second criterion, which is based on the framework orientation, permits choosing a framework depending upon the different types of studied processes. Our analysis suggests APQC and PWC-PCF-PCF are suitable for operating processes and EFQM and Baldrige for management and control processes. The third criterion, which is based on organizational levels (corporate, business unit and functional), allows the comparing and classifying of frameworks in strategic, tactic or operational terms. After the analysis, we consider that EFQM and Baldrige are related to the corporate level, and APQC-PCF and PWC-PCF are related to the functional level.

The main contribution of this paper versus others studies about identification of best practices is that it is apparently the first study to identify, analyze and classify frameworks that can support this process. This conclusion is based on the fact that no other literature was found containing a similar objective.

In the introduction we wondered where and how best practices could be found. After analyzing the aforementioned frameworks we conclude that where and how best practices are found depends on a judgment of the investigators, who based mainly on one of two hypothesis:

1 - Excellent organizations develop best practices and these practices can be identified by studying their own processes and activities.

2 - Best practices can be found in any organization, therefore practices can be identified when systematically studying each organization's processes and activities, and approaching them in context and determining the cause and effect relationship with organizational performance. For the first hypothesis, where it could be assumed that the operating processes are done very well, it is possible to pay more attention to the strategy, management and control at corporate level. Then the usage of Baldrige or EFQM would be valid to explore the most successful companies in a particular field of interest (e.g. human talent, knowledge management, product innovation, etc.). The usage of Baldrige or EFQM would also study the processes or activities (practices) that are directly related with high performance in the field of interest. This approach can be called best practices searching in Organizations of Excellence, these organizations are cataloged in this way for winning a prize or some other criteria commonly accepted. The second hypothesis could be used to identify best practices for designing new organizations in a specific sector (e.g. provision of a particular service, producing a particular good, etc.). In this case we are interested in knowing many different kinds of best practices. It is possible to adopt an approach that we could call best practices Searching in organizations (of excellence or not) with a similar business model. However, with no guarantee that all organizations studied are Organizations of Excellence, this requires a stricter consideration of the definition and demarcation of each of the activities and processes. As well as of the context in which they develop and the causal relationships they have with a desirable performance for the organization that want to 
be designed. The complexity exhibited by PWC-PCF and APQC-PCF are explicit by the precision, definition and delimitation offered, can be of great importance for the construction of a protocol for the transfer and adoption of best practices that are identified.

The main limitation of this study is that only scientific literature was analyzed, and it was found that there is an abundance of non-scientific literature about the identification of best practices. Due to this, our research focuses on the academics point of view and does not pay attention to the practitioners' point of view. We believe that future research must include the practitioner's perspective in order to evaluate and compare it. Also, we suggest extending the analysis taking into account other less widespread frameworks, such as Malaysian Quality Management Excellence Award, Singapore Quality Award Model, Japan Quality Award Model, Canadian Framework for Business Excellence and the Australian Business Excellence Framework.

\section{References}

American Productivity \& Quality Center (APQC). 2014. Process Classification Framework APQC [online], [cited 26 February 2015]. Available from Internet: http://www.apqc.org/knowledge-base/download/313690/K05162_PCF_Ver_6\%201_1.pdf

Bergek, A.; Norrman, C. 2008. Incubator best practice: a framework, Technovation 28(1-2): 20-28. http://dx.doi.org/10.1016/j.technovation.2007.07.008

European Foundation for Quality Management (EFQM). 2012a. EFQM Excellence Model 2013: Excellent Organisations achieve and sustain outstanding levels of performance that meet or exceed the expectations of all their stakeholders. Brussels, Belgium: EFQM.

European Foundation for Quality Management (EFQM). 2012b. An Overview of the EFQM Excellence Model [online], [cited 26 February 2015]. EFQM. Available from Internet: http://www. efqm.org/sites/default/files/overview_efqm_2013_v1.1.pdf

European Foundation for Quality Management (EFQM). 2009. RADARise your business for success. Brussels, Belgium: EFQM.

Harrington, H. J. 2004. The fallacy of universal best practices, Total Quality Management \& Business Excellence 15(5-6): 849-858. http://dx.doi.org/10.1080/14783360410001680288

Laugen, B.; Acur, N.; Boer, H.; Frick, J. 2005. Best manufacturing practices: what do the bestperforming companies do?, International Journal of Operations \& Production Management 25(2): 131-150. http://dx.doi.org/10.1108/01443570510577001

McCarthy, G. 2005. Leadership practices in German and UK organisations, Journal of European Industrial Training 29(3): 217-234. http://dx.doi.org/10.1108/03090590510591094

National Institute of Standards and Technology (NIST). 2013a. Baldrige Performance Excellence Program: 2013-2014 Criteria for Performance Excellence: NIST.

National Institute of Standards and Technology (NIST). 2013b. Baldrige Performance Excellence Program: 2013-2014 Education Criteria for Performance: NIST.

National Institute of Standards and Technology (NIST). 2013c. Baldrige Performance Excellence Program: 2011-2012 Health Care Criteria for Performance Excellence: NIST.

O'Leary, D. E. 2007. Empirical analysis of the evolution of a taxonomy for best practices, Decision Support Systems 43(4): 1650-1663. http://dx.doi.org/10.1016/j.dss.2006.09.004

Oakland, J.; Tanner, S.; Gadd, K. 2002. Best practice in business excellence, Total Quality Management \& Business Excellence 13(8): 1125-1139. http://dx.doi.org/10.1080/09544120200000008 
Osseo-Asare, A. E.; Longbottom, D.; Murphy, W. D. 2005. Leadership best practices for sustaining quality in UK higher education from the perspective of the EFQM Excellence Model, Quality Assurance in Education 13(2): 148-170. http://dx.doi.org/10.1108/09684880510594391

Price Waterhouse Coopers (PWC). 2011. Process classification framework [online], [cited 11 July 2011]. PWC. Available from Internet: http://globalbestpractices.pwc.com/Home/ProcessFrameworks.aspx?FW=Process+classification + framework

Saunders, M.; Mann, R.; Smith, R. 2007. Benchmarking strategy deployment practices, Benchmarking: an International Journal 14 (5): 609-623. http://dx.doi.org/10.1108/14635770710819281

Verweire, K.; Van den Berghe, L. (Eds.). 2004. Integrated performance management: a guide to strategy implementation. London, UK: Sage Publications Ltd.

Vouzas, F. K.; Gotzamani, K. D. 2005. Best practices of selected Greek organizations on their road to business excellence: the contribution of the new ISO 9000:2000 series of standards, The TQM Magazine 17(3): 259-266. http://dx.doi.org/10.1108/09544780510594225

Wellstein, B.; Kieser, A. 2011. Trading "best practices" - a good practice?, Industrial and Corporate Change 20(3): 683-719. http://dx.doi.org/10.1093/icc/dtr011

Westfall, L. 2010. The certified software quality engineer handbook. USA, Milwaukee: ASQ Quality Press.

Xu, Y.; Yeh, C. H. 2010. An optimal best practice selection approach, in 2010 Third International Joint Conference on Computational Science and Optimization, 28-31 May 2010, Huangshan, Anhui. IEEE. http://dx.doi.org/10.1109/CSO.2010.144

Gerardo Luís ANGULO CUENTAS. He is Associate Professor of the Department of Industrial Engineering at University of Magdalena (Colombia) and is $\mathrm{PhD}$ candidate in Technology Development and Management from Industrial University of Santander (Colombia). His research interests are: Innovation and Technology Management, Science and Technology Parks, and Management Practices Identification.

Jaime Alberto CAMACHO PICO. He is Associate Professor of the School of Industrial Engineering at Industrial University of Santander (Colombia) and is $\mathrm{PhD}$ in Industrial Engineering from Polytechnic University of Catalonia (Spain). His research interests are: Innovation and Technology Management, Science and Technology Parks, Economics of Technological Change, and Technological Development.

Astrid JAIME ARIAS. PhD in Industrial Engineering with emphasis on Knowledge Management and Master of Industrial Engineering, National Polytechnic Institute Grenoble - INPG (France). Specialist in Innovation and Technology Enterprise, Polytechnic University of Catalonia (Spain). Specialist Quality Management, Santo Tomas University (Colombia). Engineer Industrial, Universidad de los Andes (Colombia). Today is Director of Knowledge Transfer at Industrial University of Santander (Colombia). 